\title{
CESȦRO SUMMABILITY OF ORDINARY DOUBLE DIRICHLET SERIES
}

\author{
JOHN G. HERRIOT
}

1. Introduction. The purpose of this paper is to obtain some results in the Cesàro summability of ordinary double Dirichlet series similar to those obtained by $\mathrm{H}$. Bohr ${ }^{1}$ for the simple series. As is well known a double sequence $\left\{S_{m n}\right\}$ may tend to a finite limit as $m, n \rightarrow \infty^{2}$ without $S_{m n}$ being a bounded function of $m$ and $n$. In order to avoid difficulties in this respect and to obtain results analogous to those for simple series, the discussion will usually be restricted to bounded sequences.

Let $\sum_{m, n=1}^{\infty} u_{m n}$ be a double series of constant terms. Set

$$
\begin{aligned}
& S_{m n}^{00}=\sum_{i=1, j=1}^{m, n} u_{i j}, \quad S_{m n}^{10}=\sum_{i=1}^{m} S_{i n}^{00}, \quad S_{m n}^{01}=\sum_{j=1}^{n} S_{m j}^{00}, \\
& S_{m n}^{r s}=\sum_{i=1}^{m} S_{i n}^{r-1, s}=\sum_{j=1}^{n} S_{m j}^{r, s-1}, \quad m, n, r, s=1,2,3, \cdots .
\end{aligned}
$$

The double series is said to be summable $(C, r, s)$ with summability value $S$ if $S_{m n}^{r s} r ! s ! / m^{r} n^{s} \rightarrow S$ as $m, n \rightarrow \infty$. It is bounded $(C, r, s)$ if $S_{m n}^{r s} r ! s ! / m^{r} n^{s}$ is bounded for all $m$ and $n$. It is summable-bounded $(C, r, s)$ if it is both summable $(C, r, s)$ and bounded $(C, r, s)$. In case the $u_{m n}$ are functions of complex variables $x$ and $y$ similar definitions can be set up for uniform summability, uniform boundedness, and uniform summability-boundedness $(C, r, s)$.

G. M. Merriman ${ }^{3}$ has given the definition of summability $(C, r, s)$ of a double series in a slightly different form. But our means differ from his only by a factor which is bounded and tends to one as $m, n \rightarrow \infty$. Consequently the two definitions are equivalent.

By means of Robison's ${ }^{4}$ generalization of the Silverman-Toeplitz

${ }^{1}$ Bohr, H., Bidrag til de Dirichlet'ske Raekkers Theori, Dissertation, Copenhagen, 1910; Über die Summabilität Dirichletscher Reihen, Nachrichten von der Gesellschaft der Wissenschaften zu Göttingen, 1909, pp. 247-262; Sur la série de Dirichlet, Comptes Rendus de l'Académie des Sciences, Paris, vol. 148 (1909), pp. 75-80.

${ }^{2}$ Throughout this paper $m, n \rightarrow \infty$ means $m$ and $n$ tend to infinity simultaneously but independently.

${ }^{3}$ Merriman, G. M., $A$ set of necessary and sufficient conditions for the Cesaro summability of double series, Annals of Mathematics, (2), vol. 29 (1928), pp. 343-354.

${ }^{4}$ Robison, G. M., Divergent double sequences and series, Transactions of this Society, vol. 28 (1926), pp. 50-73 (p. 53). 
theorem, it is easily shown that summability-boundedness $(C, r-1, s)$ (or $(C, r, s-1))$ implies the same $(C, r, s)$.

In $\$ 2$ of the present paper we give some general lemmas on summable series which are mostly generalizations of theorems of Bohr. These are used in $\$ 3$ to deduce a number of interesting theorems on summability of ordinary double Dirichlet series. We define associated abscissas of summability-bounded $(C, r, s)$ and in $\$ 4$ give some theorems showing relations between them.

2. Preliminary lemmas. The following lemmas will be useful.

LEMMA 1. Let $\sum_{m, n=1}^{\infty} u_{m n}(x, y)$ be uniformly summable $(C, r, s)$ for $x$ and $y$ in every $D_{1}^{\prime}$ and $D_{2}^{\prime}$ respectively for which $D_{1}^{\prime}$ and $D_{2}^{\prime}$ are closed and contained in the domains $D_{1}$ and $D_{2}$ respectively, and let $u_{m n}(x, y)$ $(m, n=1,2,3, \cdots)$ be regular analytic functions of $x$ and $y$ for $x$ in $D_{1}$ and $y$ in $D_{2}$. Then the series represents by its summability value $S(x, y)$ an analytic function regular for $x$ in $D_{1}, y$ in $D_{2}$. Moreover the series may be differentiated partially termwise arbitrarily often for $x$ in $D_{1}$, $y$ in $D_{2}$. The series

$$
\sum_{m=1, n=1}^{\infty, \infty} \frac{\partial^{p+q} u_{m n}(x, y)}{\partial x^{p} \partial y^{q}}
$$

is summable $(C, r, s)$ for $x$ in $D_{1}, y$ in $D_{2}$, and indeed uniformly for $x$ and $y$ in every $D_{1}^{\prime}$ and $D_{2}^{\prime}$ respectively, to the function $\partial^{p+q} S(x, y) / \partial x^{p} \partial y^{q}$ $(p, q=0,1,2, \cdots)$.

LEMMA 2. Let $F_{m n}(x, y)=\sum_{i=1}^{c} v_{i}(x) \gamma_{m n i}(y)$, where $c=c(m)$ is a function of $m$ defined as a positive integer for positive integral $m$, and which increases monotonely with $m$, and let the following four conditions be satisfied:

(A) $\left|v_{i}(x)\right| \leqq v_{i}, x$ in domain $D_{1}(i=1,2,3, \cdots)$;

(B) $\sum_{i=1}^{\infty}\left|v_{i}(x)\right|$ converges uniformly in $D_{1}$;

(C) $\left|\gamma_{m n i}(y)\right| \leqq K$ (constant), $y$ in domain $D_{2}(m, n, i=1,2,3, \cdots)$;

(D) $\lim _{m, n \rightarrow \infty} \gamma_{m n i}(y)=\gamma_{i}(y)$ uniformly in $D_{2}(i=1,2,3, \cdots)$.

Then $F_{m n}(x, y)$ is uniformly bounded for $x$ in $D_{1}, y$ in $D_{2}$ for all $m$ and $n$, and, as $m, n \rightarrow \infty$, tends uniformly to the sum of the uniformly absolutely convergent series

$$
\sum_{i=1}^{\infty} v_{i}(x) \gamma_{i}(y)
$$

If the $v_{i}$ and $\gamma_{m n i}$ are constants, a similar lemma can be stated omitting all references to uniformity and dropping (A).

LEMMA 3. Let $F_{m n}(x, y)=\sum_{i, j=1}^{c, d} v_{i}(x) w_{j}(y) \gamma_{m n i j}$, where $c=c(m)$ and 
$d=d(n)$ are functions of $m$ and $n$, respectively, like $c(m)$ of Lemma 3 , and let the following conditions be satisfied:

$\left(\mathrm{A}_{1}\right)\left|v_{i}(x)\right| \leqq v_{i}, x$ in domain $D_{1}(i=1,2,3, \cdots)$;

$\left(\mathrm{A}_{2}\right)\left|w_{j}(y)\right| \leqq w_{j}, y$ in domain $D_{2}(j=1,2,3, \cdots)$;

$\left(\mathrm{B}_{1}\right) \sum_{i=1}^{\infty}\left|v_{i}(x)\right|$ converges uniformly in $D_{1}$;

$\left(\mathrm{B}_{2}\right) \sum_{j=1}^{\infty}\left|w_{j}(y)\right|$ converges uniformly in $D_{2}$;

(C) $\left|\gamma_{m n i j}\right| \leqq K$ (constant) $(m, n, i, j=1,2,3, \cdots)$;

(D) $\lim _{m, n \rightarrow \infty} \gamma_{m n i j}=\gamma_{i j}(i, j=1,2,3, \cdots)$.

Then $F_{m n}(x, y)$ is uniformly bounded for $x$ in $D_{1}, y$ in $D_{2}$ for all $m$ and $n$, and, as $m, n \rightarrow \infty$, tends uniformly to the sum of the uniformly absolutely convergent series $\sum_{i, j=1}^{\infty} v_{i}(x) w_{j}(y) \gamma_{i j}$.

Again if the $v_{i}$ and $w_{j}$ are constants a similar lemma can be stated omitting all references to uniformity and dropping (A).

We may omit the proofs of these lemmas since they are analogous to those of Bohr. ${ }^{5}$

LeMmA 4. Let $\sum_{m, n=1}^{\infty} u_{m n}$ be bounded $(C, r, s)$ and let $\left\{\alpha_{m}(x)\right\}$ and $\left\{\beta_{n}(y)\right\}$ be sequences of functions of the complex variables $x$ and $y$ which satisfy the conditions:

$\left(\mathrm{A}_{1}\right)\left|\alpha_{m}(x)\right| \leqq K_{m}, x$ in domain $D_{1}(m=1,2,3, \cdots)$;

$\left(\mathrm{A}_{2}\right)\left|\beta_{n}(y)\right| \leqq K_{n}, y$ in domain $D_{2}(n=1,2,3, \cdots)$;

$\left(\mathrm{B}_{1}\right) \sum_{m=1}^{\infty} m^{p-1}\left|\Delta^{p} \alpha_{m}(x)\right|$ converges uniformly ${ }^{6}$ in $D_{1}(p=1,2,3$, $\cdots, r+1)$;

$\left(\mathrm{B}_{2}\right) \sum_{n=1}^{\infty} n^{q-1}\left|\Delta^{q} \beta_{n}(y)\right|$ converges uniformly in $D_{2}(q=1,2,3$, ..., $s+1)$;

(C) $\lim _{m \rightarrow \infty} \alpha_{m}(x)=0, x$ in $D_{1}$;

$\left(\mathrm{C}_{2}\right) \lim _{n \rightarrow \infty} \beta_{n}(y)=0, y$ in $D_{2}$.

Then the series $\sum_{m, n=1}^{\infty} u_{m n} \alpha_{m}(x) \beta_{n}(y)$ is uniformly summable-bounded $(C, r, s)$ for $x$ in $D_{1}, y$ in $D_{2}$.

If the $\alpha_{m}$ and $\beta_{n}$ are constants, a similar lemma can be stated omitting all references to uniformity and dropping (A).

For simplicity of notation we shall indicate the proof of this simpler lemma. Let $T_{m n}^{r s}$ be formed from $\sum_{m, n=1}^{\infty} u_{m n} \alpha_{m} \beta_{n}, S_{m n}^{r s}$ from $\sum_{m, n=1}^{\infty} u_{m n}$. First we obtain the following relation between $T_{m n}^{r s}$ and $S_{m n}^{r s}$ :

$$
T_{m n}^{r s}=\sum_{p=0, q=0}^{r+1, s+1} C_{r+1, p} C_{s+1, q} P_{r, s, p, q, m, n},^{7}
$$

where

5 Bohr, loc. cit., Dissertation, pp. 53-60.

${ }^{6} \Delta^{p} \alpha_{m}=\alpha_{m}-p \alpha_{m+1}+\frac{1}{2} p(p-1) \alpha_{m+2}-\cdots+(-1)^{p} \alpha_{m+p}$.

${ }^{7} C_{r, p}$ denotes the binomial coefficient $r ! / p !(r-p)$ !. 
(3)

$$
\begin{aligned}
P_{r, s, 0,0, m, n}= & S_{m n}^{r s} \alpha_{m} \beta_{n}, \\
P_{r, s, p, 0, m, n}= & \sum_{i=1}^{m-p} S_{i n}^{r s} C_{m-i-1, p-1} \Delta^{p} \alpha_{i} \beta_{n}, p=1,2,3, \cdots, r+1, \\
P_{r, s, 0, q, m, n}= & \sum_{j=1}^{n-q} S_{m j}^{r s} C_{n-j-1, q-1} \alpha_{m} \Delta^{q} \beta_{j}, q=1,2,3, \cdots, s+1, \\
P_{r, s, p, q, m, n}= & \sum_{i=1, j=1}^{m-p, n-q} S_{i j}^{r s} C_{m-i-1, p-1} C_{n-j-1, q-1} \Delta^{p} \alpha_{i} \Delta^{q} \beta_{j}, \\
& p=1,2,3, \cdots, r+1 ; q=1,2,3, \cdots, s+1 ;
\end{aligned}
$$

if $m \leqq p$ or $n \leqq q$ or both, then $P_{r, s, p, q, m, n}=0$.

The proof is by induction on $r$ and $s$ successively making use of the relation (for induction on $r$ )

$$
\sum_{i=p+1}^{m} P_{r, s, p, q, i, n}=P_{r+1, s, p, q, m, n}+P_{r+1, s, p+1, q, m, n}
$$

Next we evaluate $\lim _{m, n \rightarrow \infty} T_{m n}^{r s} r ! s ! / m^{r} n^{s}$. By hypothesis

$$
\left|S_{m n}^{r s} r ! s ! / m^{r} n^{8}\right| \leqq K, \quad m, n=1,2,3, \cdots .
$$

Considering separately the various terms of (2) multiplied by $r ! s ! / m^{r} n^{s}$, we can show that all terms except that for which $p=r+1$, $q=s+1$ tend to zero and are bounded. For example, in case $p=1,2,3, \cdots, r+1$, and $q=0$, we use Lemma 2 (modified) choosing

$$
c=m-p, v_{i}=i^{p-1} \Delta^{p} \alpha_{i}
$$

and taking up the remaining factors in $\gamma_{m n i}$. Then $\gamma_{m n i} \rightarrow 0$ as $m, n \rightarrow \infty$ and conditions (B), (C), and (D) of Lemma 2 are satisfied. In case $p=1,2,3, \cdots, r+1$ and $q=1,2,3, \cdots, s+1$, we apply Lemma 3 (modified). The term for which $p=r+1, q=s+1$ is thus seen to be bounded and to tend to a finite limit not necessarily zero as $m, n \rightarrow \infty$. This completes the proof of the (modified) lemma.

3. Double Dirichlet series. We now make use of the lemmas of $\S 2$ to prove some theorems concerning the summability of the ordinary double Dirichlet series

$$
\sum_{m=1, n=1}^{\infty, \infty} a_{m n} / m^{x} n^{y}
$$

Theorem 1. If the double Dirichlet series (5) is bounded $(C, r, s)$ for 
the place $\left(x_{0}, y_{0}\right)$, then it is summable-bounded $(C, r, s)$ for every place $(x, y)$ for which $R(x)>R\left(x_{0}\right)$ and $R(y)>R\left(y_{0}\right) .^{9}$

Proof. Let $x=x_{0}+\delta, y=y_{0}+\eta, R(\delta)>0, R(\eta)>0$. We apply Lemma 4 (modified) taking

$u_{m n}=a_{m n} / m^{x_{0}} n^{y_{0}}, \quad \alpha_{m}=1 / m^{\delta}, \quad \beta_{n}=1 / n^{\eta}, \quad u_{m n} \alpha_{m} \beta_{n}=a_{m n} / m^{x} n^{y}$.

Then $\lim _{m \rightarrow \infty} \alpha_{m}=\lim _{n \rightarrow \infty} \beta_{n}=0$. Also, using the method of Bohr, ${ }^{10}$

$$
\begin{array}{rl}
\Delta^{p} \alpha_{m}=\delta(\delta+1) \cdots(\delta+p-1) \int_{m}^{m+1} & d a_{1} \int_{a_{1}}^{a_{1}+1} d a_{2} \cdots \\
& \cdot \int_{a_{p-2}}^{a_{p-1+1}} d a_{p-1} \int_{a_{p-1}}^{a_{p-1+1}} \frac{d a_{p}}{a_{p}^{p+\delta}} .
\end{array}
$$

Hence $m^{p-1}\left|\Delta^{p} \alpha_{m}\right| \leqq|\delta||\delta+1| \cdots|\delta+p-1| \cdot 1 / m^{1+R(\delta)}(p=1,2,3$, $\cdots, r+1)$, so that condition $\left(\mathrm{B}_{1}\right)$ of Lemma 4 is satisfied. Likewise $\left(B_{2}\right)$ is satisfied. By the remark following Lemma 4, Theorem 1 follows.

Even if (5) is summable-bounded $(C, r, s)$ for the place $\left(x_{0}, y_{0}\right)$, it does not necessarily follow that it is summable-bounded $(C, r, s)$ for every place $\left(x_{0}, y\right)$ for which $R(y)>R\left(y_{0}\right)$, or for every place $\left(x, y_{0}\right)$ for which $R(x)>R\left(x_{0}\right)$. For let $r=s=0$ and let $a_{11}=-1$, $a_{1 n}=1 / n(n-1)(n=2,3,4, \cdots), a_{m 1}=2(-1)^{m}(m=2,3,4, \cdots)$, $a_{m n}=2(-1)^{m-1} / n(n-1)(m, n=2,3,4, \cdots)$. Then $S_{m n}^{00}=(-1)^{m} / n$ $(m, n=1,2,3, \cdots)$. Hence for $x=y=0$ the series (5) is convergentbounded with sum zero. But for $x=0, y=1$ the series fails to converge for

$$
T_{m n}^{00}=\sum_{i=1, j=1}^{m, n} \frac{a_{i j}}{i^{0} j^{1}}=(-1)^{m} \sum_{j=1}^{n-1} \frac{1}{j^{2}(j+1)}+\frac{(-1)^{m}}{n^{2}},
$$

which tends to no limit as $m, n \rightarrow \infty$.

From Theorem 1 we deduce the existence of associated abscissas of summability-bounded $(C, r, s)$, that is, numbers $\lambda_{r}, \mu_{s}$ such that (5) is summable-bounded $(C, r, s)$ in the associated domains $R(x)>\lambda_{r}$, $R(y)>\mu_{s}$ and not summable-bounded $(C, r, s)$ in the associated domains $R(x)<\lambda_{r}, R(y)<\mu_{s}$.

${ }^{8} R(x)$ denotes the real part of $x$.

9 This theorem and Theorems 3 and 7 bear some resemblance to but are not the same as Theorem IV of Merriman, Concerning the summability of double series of $a$ certain type, Annals of Mathematics, (2), vol. 28 (1927), pp. 515-533. Cf. M. Gurney, Cesaro summability of double series, this Bulletin, vol. 38 (1932), pp. 825-827.

10 Bohr, loc. cit., Nachrichten von der Gesellschaft der Wissenschaften zu Göttingen, pp. 248-249. 
The question arises whether the series (5) has associated abscissas of summability $(C, r, s)$ (the condition of boundedness not being imposed), that is, whether there exists a pair of numbers $\lambda_{r}^{\prime}, \mu_{s}^{\prime}$ such that the series is summable $(C, r, s)$ at all $(x, y)$ for which $R(x)>\lambda_{r}^{\prime}$, $R(y)>\mu_{s}^{\prime}$, and is not summable $(C, r, s)$ at any $(x, y)$ for which $R(x)<\lambda_{r}^{\prime}, R(y)<\mu_{s}^{\prime}$. The answer is not in general in the affirmative. The following is an example of a series which is convergent but unbounded at $x=y=0$, but at all other places it fails to converge and at all places it is not summable $(C, r, s)$ for any positive integral $r$ and $s$. Let $a_{1 n}=-a_{2 n}=n^{n}(n=1,2,3, \cdots), a_{m 1}=-a_{m 2}=m^{m}$ $(m=3,4,5, \cdots), a_{m n}=0(m, n=3,4,5, \cdots)$. Then

$S_{m n}^{00}=\left(1-2^{-x}\right) \sum_{j=1}^{n} j^{j-y}+\left(1-2^{-y}\right) \sum_{i=3}^{m} i^{i-x}, \quad m, n=3,4,5, \cdots$.

If $x=y=0$, these $S_{m n}^{00}$ are all 0 , but otherwise the required limit does not exist. The evaluation of $S_{m n}^{r s}$ is too involved to give here, but for all $r>0, s>0$, all $(x, y), S_{m n}^{r s} r ! s ! / m^{r} n^{s}$ tends to no finite limit as $m, n \rightarrow \infty$.

That such behavior is exceptional is shown by

Theorem 2. If the double Dirichlet series (5) is summable $(C, r, s)$ in a domain $D$ of the two complex variables $x$ and $y$, it is bounded $(C, r, s)$ at each point of this domain.

Leja ${ }^{11}$ has given a similar theorem concerning the convergence of general double Dirichlet series. Our theorem may be proved by a method analogous to his. The first step is to show that if the series (5) is summable $(C, r, s)$ at the points $\left(x_{0}, y\right)$, where $x_{0}$ is fixed and $y$ runs over a plane domain $d$, then, for $j=1,2,3, \cdots$, the series $\sum_{i=1}^{\infty} a_{i j} / i^{x_{0}}$ are bounded $(C, r)$.

Theorem 3. A double Dirichlet series (5) having a pair of associated abscissas of summability-bounded $(C, r, s) \lambda_{r}, \mu_{s}$ is uniformly summablebounded $(C, r, s)$ for $x$ and $y$ in every pair of associated regions $D_{1}$ and $D_{2}$ defined by the relations $D_{1}: R(x) \geqq \lambda_{r}+\epsilon,|x| \leqq E ; D_{2}: R(y) \geqq \mu_{s}+\epsilon$, $|y| \leqq E, \epsilon$ and $E$ being arbitrary finite positive numbers.

Proof. Let $x_{0}=\lambda_{r}+\epsilon / 2, y_{0}=\mu_{s}+\epsilon / 2$, and let $x$ be in $D_{1}, y$ in $D_{2}$. The proof is now similar to that of Theorem 1 except that we apply Lemma 4 as stated.

THEOREM 4. If $\lambda_{r}, \mu_{s}$ are a pair of associated abscissas of summabil-

${ }^{11}$ Leja, F., Sur les séries de Dirichlet doubles, Comptes-Rendus du 1mier Congrès des Mathématiciens des Pays Slaves, Warsaw, 1929, pp. 140-158. 
ity-bounded $(C, r, s)$ of the double Dirichlet series

$$
\sum_{m=2, n=2}^{\infty, \infty} a_{m n} / m^{x} n^{y}
$$

then $\lambda_{r}, \mu_{s}$ are also a pair of associated abscissas of summabilitybounded $(C, r, s)$ of

$$
\sum_{m=2, n=2}^{\infty, \infty} a_{m n}(\log m)^{\alpha}(\log n)^{\beta} / m^{x} n^{y}=\sum_{m=2, n=2}^{\infty, \infty} b_{m n} / m^{x} n^{y},
$$

where $\alpha$ and $\beta$ are arbitrary complex numbers, and conversely.

Proof. Clearly it suffices to show that, if (6) is summable-bounded $(C, r, s)$ for $\left(x_{0}, y_{0}\right)$, then (7) is summable-bounded $(C, r, s)$ at every $(x, y)$ for which $R(x)>R\left(x_{0}\right)$ and $R(y)>R\left(y_{0}\right)$. To prove this we take $x=x_{0}+\delta, y=y_{0}+\eta, R(\delta)>0, R(\eta)>0$, and apply Lemma 4 (modified) with

$$
\begin{aligned}
& u_{m n}=a_{m n} / m^{x_{0}} n^{y_{0}}, \quad \alpha_{m}=(\log m)^{\alpha} / m^{\delta}, \quad \beta_{n}=(\log n)^{\beta} / n^{\eta}, \\
& u_{m n} \alpha_{m} \beta_{n}=b_{m n} / m^{x} n^{y} \text {. }
\end{aligned}
$$

Then $\sum_{m, n=2}^{\infty} u_{m n}$ is summable-bounded $(C, r, s)$ and condition $(\mathrm{C})$ is satisfied. Again, using the method of $\mathrm{Bohr}^{12}$

$$
\begin{aligned}
& \Delta^{p} \alpha_{m}=(-1)^{p} \int_{m}^{m+1} d a_{1} \int_{a_{1}}^{a_{1}+1} d a_{2} \ldots \\
& \cdot \int_{a_{p-2}}^{a_{p-2}+1} d a_{p-1} \int_{a_{p-1}}^{a_{p-1}+1} \frac{d^{p}}{d a_{p}^{p}} \frac{\left(\log a_{p}\right)^{\alpha}}{a_{p}^{\delta}} d a_{p}, \\
& \quad p=1,2,3, \cdots, r+1 .
\end{aligned}
$$

Now the integrand in the last integral is easily shown to be the sum of a finite number of terms of the form a constant times $\left(\log a_{p}\right)^{\gamma} / a_{p}^{p+\delta}$. Also $\left(\log a_{p}\right)^{\gamma} / a_{p}^{R(\delta) / 2}$ is bounded for $a_{p} \geqq 2$. Hence $m^{p-1}\left|\Delta^{p} \alpha_{m}\right|$ is not greater than a constant times $1 / m^{1+R(\delta) / 2}$, so that condition $\left(\mathrm{B}_{1}\right)$ and similarly $\left(B_{2}\right)$ of Lemma 4 are satisfied and the desired result follows at once.

TheOREM 5. A series (5) having a pair of associated abscissas of summability-bounded $(C, r, s) \lambda_{r}, \mu_{s}$ represents by its summability value an analytic function $f(x, y)$ regular in the associated half-planes $R(x)>\lambda_{r}, R(y)>\mu_{s}$. The series may be differentiated partially termwise arbitrarily often in these half-planes, that is, the double Dirichlet series

12 Bohr, loc. cit., Dissertation, p. 83. 


$$
\sum_{m=1, n=1}^{\infty, \infty} a_{m n}(-\log m)^{p}(-\log n)^{q} / m^{x} n^{y}
$$

is again summable-bounded $(C, r, s)$ in the same associated half-planes to the function $\partial^{p+q} f(x, y) / \partial x^{p} \partial y^{q}(p, q=0,1,2, \cdots)$.

This theorem follows immediately from Theorems 3 and 4 and Lemma 1.

A theorem similar to Theorem 5 can be stated and proved for termwise integration of (5) within its domain of summability-bounded.

THEOREM 6. The analytic function defined by the summability value $(C, r, s)$ of $(5)$ forms the analytic continuation of the analytic function defined by the sum of the series in a pair of associated half-planes, $R(x)>\lambda_{0}, R(y)>\mu_{0}$, of convergence-bounded (provided that $\lambda_{0}, \mu_{0}$ are not also associated abscissas of summability-bounded $(C, r, s))$.

This is clear from the consistency of the Cesàro mean transformation.

THEOREM 7. If the series (5) is bounded $(C, r+1, s)$ for $\left(x_{0}, y_{0}\right)$, then it is summable-bounded $(C, r, s)$ for every $(x, y)$ for which $R(x)>R\left(x_{0}\right)+1$ and $R(y)>R\left(y_{0}\right)$.

Proof. Let $x=x_{0}+1+\delta, y=y_{0}+\eta, R(\delta)>0, R(\eta)>0$. Set $u_{m n}=a_{m n} / m^{x_{0}} n^{y_{0}}, \quad \alpha_{m}=1 / m^{1+\delta}, \quad \beta_{n}=1 / n^{\eta}, \quad u_{m n} \alpha_{m} \beta_{n}=a_{m n} / m^{x} n^{y}$. Let $S_{m n}^{r s}$ be formed from $\sum_{m, n=1}^{\infty} u_{m n}, T_{m n}^{r s}$ from (5). Then

$$
\left|S_{m n}^{r+1, s}(r+1) ! s ! / m^{r+1} n^{s}\right| \leqq K, \quad m, n=1,2,3, \cdots .
$$

Since $S_{m n}^{r s}=S_{m n}^{r+1, s}-S_{m-1, n}^{r+1 . s}$, we have

$$
\begin{array}{r}
\left|\frac{S_{m n}^{r s} r ! s !}{m^{r+1} n^{s}}\right| \leqq\left|\frac{\left(\left|S_{m n}^{r+1, s}\right|+\left|S_{m-1, n}^{r+1, s}\right|\right)(r+1) ! s !}{m^{r+1} n^{s}(r+1)}\right| \leqq 2 K /(r+1), \\
m, n=1,2,3, \cdots .
\end{array}
$$

We have to prove that $T_{m n}^{r s} r ! s ! / m^{r} n^{s}$ is bounded for all $m$ and $n$ and tends to a finite limit as $m, n \rightarrow \infty$. The proof is now similar to that of Lemma 4 except that in applying Lemmas 2 and 3 we take $v_{i}=i^{p} \Delta^{p} \alpha_{i}$ instead of $i^{p-1} \Delta^{p} \alpha_{i}$.

COROLlARY. If $\lambda_{r}, \mu_{s}$ are a pair of associated abscissas of summability-bounded $(C, r, s)$ of (5), there exists a pair of associated abscissas of summability-bounded $(C, r+1, s) \lambda_{r+1}, \mu_{s}$ such that $0 \leqq \lambda_{r}-\lambda_{r+1} \leqq 1$. 
4. Associated abscissas of summability-bounded. Leja ${ }^{13}$ has given a number of theorems showing the relationship between the associated abscissas of convergence-bounded of general double Dirichlet series. We now generalize the content of these theorems for the case of ordinary double Dirichlet series to obtain relations between the associated abscissas of summability-bounded $(C, r, s)$ of the series (5). For simplicity of notation we set

$$
A=\limsup _{m+n \rightarrow \infty} \frac{\log \left|S_{m n}^{r s} r ! s ! / m^{r} n^{s}\right|}{\lambda_{r} \log m+\mu_{s} \log n},
$$

where $S_{m n}^{r s}$ is formed from $\sum_{m, n=1}^{\infty} a_{m n}$.

THEOREM 8. If $\lambda_{r}>0$ and $\mu_{s}>0$ and if $A \leqq 1$, then the series (5) is summable-bounded $(C, r, s)$ for every $(x, y)$ for which $R(x)>\lambda_{r}$ and $R(y)>\mu_{s}$.

Proof. Let $x=\lambda_{r}+\delta, y=\mu_{s}+\eta, R(\delta)>0, R(\eta)>0$. Choose $\epsilon>0$ but less than the minimum of $R(\delta) / 2 \lambda_{r}$ and $R(\eta) / 2 \mu_{s}$. Then there exists $K(\epsilon)$ such that

$$
\left|S_{m n}^{r s} r ! s ! / m^{r} n^{8}\right|<K m^{\lambda_{r}(1+\epsilon)} n^{\mu_{s}(1+\epsilon)}, \quad m, n=1,2,3, \cdots .
$$

Form $T_{m n}^{r s}$ from (5) and set

$$
u_{m n}=a_{m n}, \quad \alpha_{m}=1 / m^{x}, \quad \beta_{n}=1 / n^{y}, \quad u_{m n} \alpha_{m} \beta_{n}=a_{m n} / m^{x} n^{y} .
$$

It suffices to prove $T_{m n}^{r s} r ! s ! / m^{r} n^{s}$ is bounded for all $m$ and $n$ and tends to a finite limit as $m, n \rightarrow \infty$. The proof is now similar to that of Lemma 4 except that in applying Lemmas 2 and 3 we take $v_{i}=i^{p-1} \Delta^{p} \alpha_{i} i^{\lambda_{r}+R(\delta) / 2}$ instead of $i^{p-1} \Delta^{p} \alpha_{i}$.

THEOREM 9. If $\lambda_{r}>0$ and $\mu_{s}>0$ and if the series (5) is summablebounded $(C, r, s)$ for all $(x, y)$ for which $R(x)>\lambda_{r}$ and $R(y)>\mu_{s}$, then $A \leqq 1$.

Proof. Let any $\epsilon>0$ be assigned. Then (5) is summable-bounded $(C, r, s)$ for $x=\lambda_{r}(1+\epsilon / 2), y=\mu_{s}(1+\epsilon / 2)$. Set

$$
u_{m n}=a_{m n} / m^{x} n^{y}, \quad \alpha_{m}=m^{x}, \quad \beta_{n}=n^{y}, \quad u_{m n} \alpha_{m} \beta_{n}=a_{m n} .
$$

Forming $S_{m n}^{r s}$ from $\sum_{m, n=1}^{\infty} a_{m n}$ and $T_{m n}^{r s}$ from $\sum_{m, n=1}^{\infty} u_{m n}$ and interchanging $T_{m n}^{r s}$ and $S_{m n}^{r s}$ in (2) we obtain finally

$$
\left|\frac{S_{m n}^{r s} r ! s !}{m^{r} n^{s}}\right| \leqq \frac{r ! s !}{m^{r} n^{s}} \sum_{p=0, q=0}^{r+1, s+1} C_{r+1, p} C_{s+1, q}\left|P_{r, s, p, q, m, n}\right|
$$

\footnotetext{
${ }^{13}$ Leja, F., loc. cit.
} 
where now the $P_{r, s, p, q, m, n}$ involve the $T_{i j}^{r s}$ in place of the $S_{i j}^{r s}$. Since $\left|T_{m n}^{r s} r ! s ! / m^{r} n^{s}\right| \leqq K$ (constant) $(m, n=1,2,3, \cdots)$, it is easily shown that each term of the right member of (8) is dominated by $K^{\prime} m^{x} n^{y}{ }^{14}$ Hence

$$
\left|S_{m n}^{r s} r ! s ! / m^{r} n^{s}\right| \leqq K^{\prime \prime} m^{x} n^{y}=K^{\prime \prime} m^{\lambda_{r}(1+\epsilon / 2)} n^{\mu_{s}(1+\epsilon / 2)} .
$$

Consequently there exists $N$ such that for all $m, n$ for which $m+n>N$

$$
\left|S_{m n}^{r s} r ! s ! / m^{r} n^{*}\right| \leqq m^{\lambda_{r}(1+\epsilon)} n^{\mu_{s}(1+\epsilon)},
$$

from which the theorem follows.

TheOREM 10. A necessary and sufficient condition that $\lambda_{r}, \mu_{s}(>0)$ be a pair of associated abscissas of summability-bounded $(C, r, s)$ of the series (5) is that $A=1$.

This theorem is an immediate consequence of Theorems 8 and 9. BROWN UNIVERSITY

14 The proof of this is analogous to that of Bohr, loc. cit., Dissertation, pp. 92-93. 